\title{
LBQS 0103-2753: A BINARY QUASAR IN A MAJOR MERGER
}

\author{
G. A. Shields ${ }^{1}$, D. J. Rosario ${ }^{2}$, V. JunkKarinen ${ }^{3}$, S. C. Chapman ${ }^{4}$, E. W. Bonning ${ }^{5}$, and T. Chiba ${ }^{6}$ \\ ${ }^{1}$ Department of Astronomy, University of Texas, Austin, TX 78712, USA; shields@ astro.as.utexas.edu \\ ${ }^{2}$ Max Planck Institute for Extraterrestrial Physics, Garching 85748, Germany; rosario@mpe.mpg.de \\ ${ }^{3}$ CASS, University of California, San Diego, La Jolla, CA 92093, USA; vesa@ucsd.edu \\ ${ }^{4}$ Institute of Astronomy, University of Cambridge, Madingley Road, Cambridge CB3 0HA, UK; schapman@ast.cam.ac.uk \\ 5 YCAA, Department of Physics, Yale University, New Haven, CT 06520, USA; erin.bonning@yale.edu \\ ${ }^{6}$ Department of Applied Physics, Yale University, New Haven, CT 06520, USA; tamara.chiba@yale.edu \\ Received 2011 August 27; accepted 2011 November 8; published 2011 December 22
}

\begin{abstract}
We present Hubble Space Telescope (HST) and United Kingdom Infrared Telescope spectra and images of the $2 \mathrm{kpc}\left(0^{\prime \prime} .3\right)$ binary quasar LBQS 0103-2753 $(z=0.858)$. The HST images ( $V$ and $I$ bands) show tidal features demonstrating that this system is a major galaxy merger in progress. A two-color composite image brings out knots of star formation along the tidal arc and elsewhere. The infrared spectrum shows that both objects are at the same redshift and that the discrepant redshift of $\mathrm{C}$ IV in component $\mathrm{A}$ is not representative of the true systemic redshift of this component. LBQS 0103-2753 is one of the most closely spaced binary QSOs known and is one of the relatively few dual active galactic nuclei showing confirmed broad emission lines from both components. While statistical studies of binary QSOs suggest that simultaneous fueling of both black holes during a merger may be relatively rare, LBQS 0103-2753 demonstrates that such fueling can occur at high luminosity at a late stage in the merger at nuclear spacing of only a few kpc, without severe obscuration of the nuclei.
\end{abstract}

Key words: black hole physics - galaxies: active - quasars: general

Online-only material: color figures

\section{INTRODUCTION}

Dual active galactic nuclei (AGNs), representing merging galaxies with both nuclei in an active state, are a subject of increasing interest. Dual broad-line AGNs (BLAGNs) at spacings of 10-100 kpc, color-selected and spatially resolved from the ground, have been thought to occur with a frequency of about 1 per thousand BLAGN (Kochanek 1999; Hennawi 2006). However, a recent study by Liu et al. (2011) finds a frequency as much as 10 times higher than this for a sample consisting primarily of narrow-line AGNs in the Sloan Digital Sky Survey (SDSS). ${ }^{7}$ Several more closely spaced dual AGNs are known from a variety of observational techniques. Hubble Space Telescope (HST) imaging has revealed LBQS 0103-2753 at $2 \mathrm{kpc}$ (Junkkarinen et al. 2001) and COSMOS 100043.15+020637.2 at a similar spacing (Comerford et al. 2009; Civano et al. 2011). A close pair observed in X-rays is the ULIRG NGC 6240 at a spacing of $1.4 \mathrm{kpc}$ (Komossa 2003). The closest known binary black hole, spaced by $7 \mathrm{pc}$, is in the radio galaxy $0402+379$ (Rodriguez et al. 2009). Junkkarinen et al. (2001) estimated a frequency of 1 dual QSO per 1000 QSOs at spacings $\sim 2 \mathrm{kpc}$. They argued that this was surprisingly low if one assumes that most luminous AGNs are triggered by mergers and that fueling of one black hole is often accompanied by fueling of the other. A similar conclusion was reached for spatially resolved dual AGNs in a theoretical study by Foreman et al. (2009). In the last several years, a number of studies of dual AGNs have appeared, including systematic searches for candidate objects based on spectroscopic indicators (Wang et al. 2009; Liu et al. 2010b; Smith et al. 2010) and follow-up imaging and spectroscopic studies (Liu et al. 2010a; Fu et al. 2011; Rosario et al. 2011). A detailed study of a $z=0.44$ dual quasar with a spacing

\footnotetext{
7 The SDSS Web site is http://www.sdss.org.
}

of $21 \mathrm{kpc}$ is given by Green et al. (2010) and X-ray confirmation of a dual AGN at $2 \mathrm{kpc}$ spacing was recently reported by Comerford et al. (2011). A majority of these objects show only narrow emission lines. Closely spaced $(<10 \mathrm{kpc})$ dual AGNs showing broad emission lines from both components, as does LBQS 0103-2753, are relatively rare.

The binary nature of LBQS 0103-2753 was discovered by Junkkarinen et al. (2001) in the course of ultraviolet spectroscopic observations with Space Telescope Imaging Spectrograph (STIS) aimed at understanding the broad absorption lines. The acquisition image revealed two AGNs separated by 0.3 arcsec with magnitude $V=18.2$ and 19.4 for components $\mathrm{A}$ and $\mathrm{B}$, respectively. However, the acquisition image was too shallow to show the host galaxies. Moreover, there was an apparent discrepancy between the redshifts of the two components. Junkkarinen et al. assumed that this resulted from the presence of BAL features in the brighter component, which can affect the redshift of the C IV emission line used for the redshift. In this paper, we present follow-up imaging and spectroscopic observations that confirm this exceptional object as a true dual AGN in an ongoing galactic merger.

\section{OBSERVATIONS}

We have obtained imaging of LBQS 0103-2753 aimed at revealing the nature of the host galaxy and spectroscopy aimed at clarifying the redshift agreement between components $\mathrm{A}$ and $\mathrm{B}$ and testing for variability of the BAL features of component $\mathrm{A}$.

\subsection{HST Images}

LBQS 0107-2753 was imaged with the Advanced Camera for Surveys on HST using the F606W and F814W filters for the purpose of studying the host galaxy morphology and any merger signatures (GO 9498, PI: Junkkarinen). These filters correspond roughly to rest wavelengths of 3300 and $4400 \AA$, spanning the 

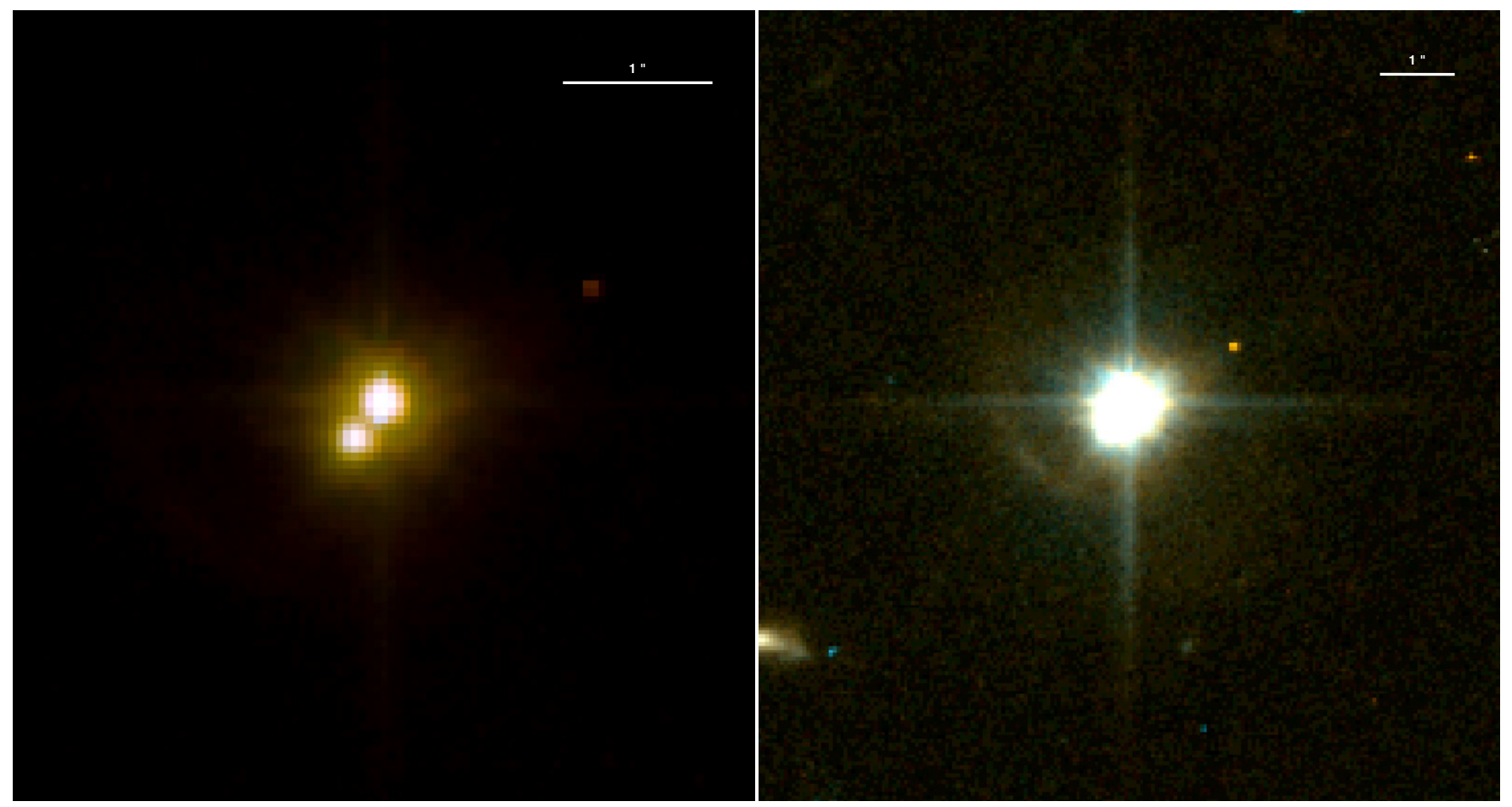

Figure 1. Two-color image of LBQS 0103-2753 representing a combination of F606W and F814W exposures with the ACS on HST. The left panel gives a shallower rendition at a larger spatial scale to display the two QSOs. The right panel gives a deeper image to bring out the host galaxy and tidal arc. See the text for discussion. (A color version of this figure is available in the online journal.)

break at $\mathrm{Ca}$ II $\mathrm{H}$ and $\mathrm{K}$. Each image is composed of four dithered subexposures, each of $1020 \mathrm{~s}$, yielding a total integration time of $4080 \mathrm{~s}$ per filter. A four-point box dither was used, with a box size of 0.265 arcsec. The images were combined using the standard version of MULTIDRIZZLE run by the archive pipeline. Remaining cosmic rays (CRs) were removed using the CRREJECT task in IRAF. ${ }^{8}$ A conservative approach was employed in the CR rejection algorithm so as not to remove flux from the bright QSO point-source cores. Therefore, some weak $\mathrm{CR}$ residuals still remain in the final image.

Figure 1 shows a two-color rendition of the HST images. The left panel brings out the two AGNs. The right panel is zoomed out a factor of two and gives a deeper rendition, clearly showing the host galaxy. There is a prominent tidal arc at about $10 \mathrm{kpc}$ from the nucleus that can be traced from position angles roughly $30^{\circ}-180^{\circ}$, reaching a distance of $\sim 15 \mathrm{kpc}$ at the northeast extreme. A possible companion galaxy is visible 6 arcsec SE of the quasar, but the full image does not indicate the presence of a rich cluster.

In an effort to bring out the host galaxy morphology, the light of the two quasars was subtracted using a point-spread function (PSF) derived from neighboring stars in each image. Figure 2 shows a difference image with F606W in blue and F814W in yellow, made with the PSF-subtracted images. This brings out several regions of intense star formation that are strong in the shorter wavelength exposure, including the main tidal arc, a smaller counterarc, and some other scattered patches with a bluer stellar population. The tidal features and disturbed morphology confirm that the system is a major merger in progress. The tidal arm structure is somewhat reminiscent of

\footnotetext{
8 IRAF is distributed by the National Optical Astronomy Observatory, which is operated by the Association of Universities for Research in Astronomy, Inc., under cooperative agreement with the National Science Foundation.
}

NGC 4038/39 (the "Antennae"); however, that system has a nuclear spacing of $\sim 6 \mathrm{kpc}$ (Stanford et al. 1990), about three times larger than for LBQS 0103-2753.

There is a diffuse envelope of starlight visible to radii $\sim 20-25 \mathrm{kpc}$ from the nucleus. In order to study the light profile of this envelope, we performed annular photometry on the $I$-band image in the radial range from 0.5 to 4.0 . The profile is well fitted by a Sérsic function with an index of 0.6 and half-light radius of 1 1".8 $(14 \mathrm{kpc})$. This very flat profile is suggestive of a disk but might also represent a distended envelope resulting from the merger. An integration of the best-fit profile over all radii gives a total flux in $\mathrm{F} 814 \mathrm{~W}$, which translates to an absolute blue magnitude of $M_{B}=-23.0$. This is about $10 \%$ of the total light from the QSOs.

\subsection{Infrared Image}

In the course of acquiring infrared spectra with United Kingdom Infrared Telescope (UKIRT, see below), we obtained a $K$-band image in approximately 0.5 arcsec seeing. The image is elongated in the position angle of the binary AGN, showing that the two nuclei are marginally resolved. Modeling the image with two-point sources based on the PSF of nearby stars gives magnitudes of $K=16.46$ and 17.85 for components $\mathrm{A}$ and $\mathrm{B}$, respectively, with an uncertainty of about $0.1 \mathrm{mag}$. Absent brightness variations since the Junkkarinen et al. (2001) observations, give colors of $V-K=-1.7$ and -1.6 for $\mathrm{A}$ and $\mathrm{B}$, respectively, so that there is little difference in optical-infrared color between the two components.

\subsection{Infrared Spectrum}

Junkkarinen et al. (2001) found a discrepancy of $\sim 4000 \mathrm{~km} \mathrm{~s}^{-1}$ between the redshifts of components A and B, based on the centroid of the C IV $1549 \AA$ emission-line profile 


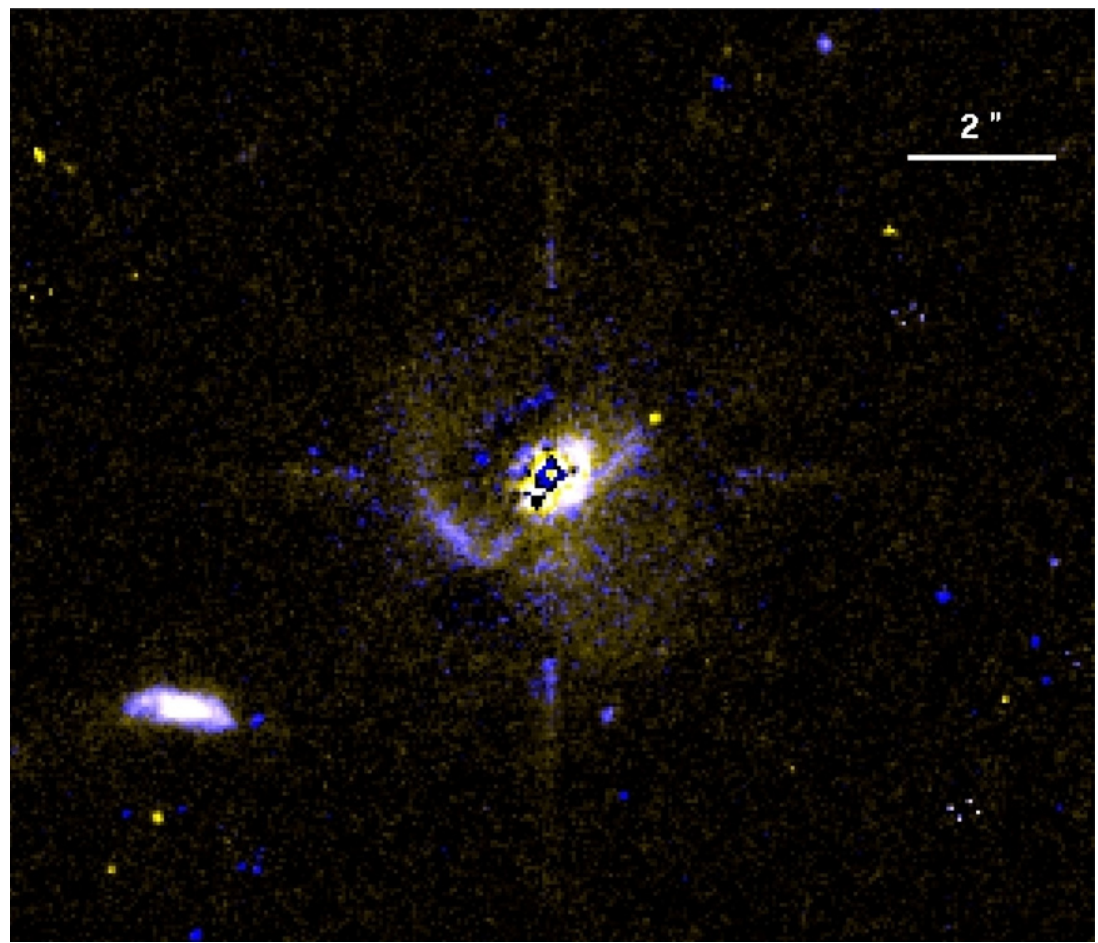

Figure 2. PSF-subtracted two-color image of LBQS 0103-2753 weighted to bring out the regions of recent star formation in the main tidal arc and the counterarc. See the text for discussion.

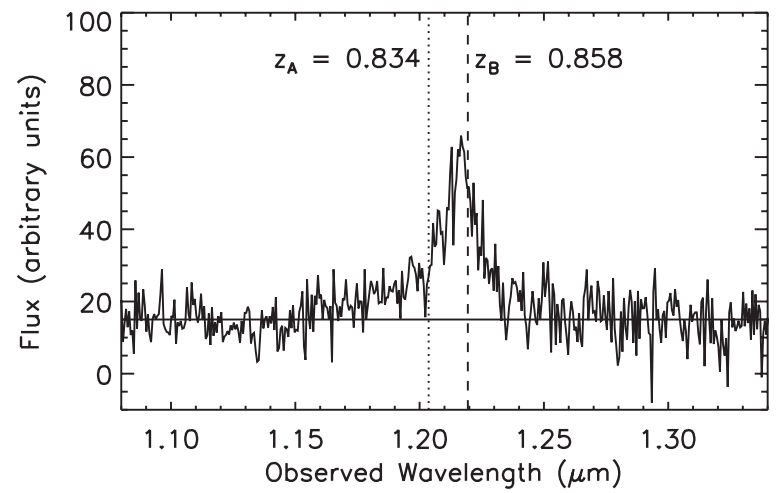

Figure 3. Infrared $J$-band spectrum of LBQS 0103-2753 obtained with UKIRT. The expected wavelengths of $\mathrm{H} \alpha$ for the redshift of the $\mathrm{C}$ IV peak in component $\mathrm{A}$ $(z=0.834)$ and of component $\mathrm{B}(z=0.858)$ are shown. There is no indication of a feature at $z_{\mathrm{A}}$, confirming that the $\mathrm{C}$ IV peak in component $\mathrm{A}$ is affected by the BAL absorption and does not represent the true redshift. See the text for discussion.

above $50 \%$ of the peak flux level $\left(z_{A}=0.834\right.$ and $\left.z_{B}=0.858\right)$. They noted, however, that the C IV emission-line peak can be substantially skewed in BAL QSOs (Richards et al. 2003). The $\mathrm{H} \alpha$ emission line should not be affected by the BALs. In order to test for the redshift agreement of components A and B, we obtained a $J$-band infrared spectrum with the UKIRT on the night of 2001 August 28. The setup employed the CGS4 spectrograph with a grating of 40 lines per $\mathrm{mm}$ and a 2 pixel slit $(1.2$ arcsec), giving resolution $\mathrm{R} / 400$. The detector was a $256 \times 256$ array. A total of 12 exposures of $60 \mathrm{~s}$ were obtained using alternate chops along the slit. Standard calibration procedures were carried out using the XIDL package (http://www.ucolick.org/ xavier/IDL).

The reduced $J$-band spectrum is shown in Figure 3. This spectrum includes the light from both components of the dual QSO. The broad $\mathrm{H} \alpha$ line is evident. The profile suggests a single redshift rather than a superposition of two lines at the $C$ IV values of $z_{A}$ and $z_{B}$. The wavelength of the $\mathrm{H} \alpha$ peak is $12175 \AA$, giving a redshift of $z=0.855 \pm 0.004$ for the combined $\mathrm{H} \alpha$ profile. The error is based on comparing the centroid at different heights in the line profile above half-maximum and is also consistent with extreme visual cursor settings. This redshift is in agreement with $z_{B}=0.858$. The expected wavelength of $\mathrm{H} \alpha$ for redshift $z_{A}$ is $12036 \AA$, which is distinctly displaced to the blue wing of the observed profile. Since component $\mathrm{A}$ is the brighter component, one would expect the combined $\mathrm{H} \alpha$ profile to be dominated by component $\mathrm{A}$. Thus, if the $\mathrm{C}$ IV value of $z_{A}$ represented the true redshift, a prominent peak at this redshift should be evident in the $\mathrm{H} \alpha$ profile. We conclude that the $\mathrm{C}$ IV redshift of component $\mathrm{A}$ is misleading and that the true redshift of component $\mathrm{A}$ agrees with component B. In view of the low probability of a chance superposition (Junkkarinen et al. 2001) and the tidal features evident in the images, we conclude that this system is indeed a dual AGN in an ongoing merger.

Taking $z_{B}$ as a reference, the $\mathrm{C}$ IV offset for component $\mathrm{A}$ is unusually large. Most C IV offsets are less than $2000 \mathrm{~km} \mathrm{~s}^{-1}$ (Richards et al. 2011). This remains true for BAL QSOs according to the analysis by Richards et al. using the "blueshift" of the C III]-SI III] blend as a predictor of the C IV offset. This predictor is difficult to apply in LBQS 0103-2753 because of the strong, blended $\mathrm{Al}$ III feature (see below).

The quality of the H $\alpha$ spectrum is not sufficient to resolve the two components for a typical merger line-of-sight velocity of a few hundred $\mathrm{km} \mathrm{s}^{-1}$, and we cannot assess the actual orbital velocity. The significance of the $\mathrm{H} \alpha$ spectrum is simply to remove the C IV value of $z_{A}$ as a concern for the dual quasar interpretation.

\subsection{HST Spectra}

Spectra of LBQS 0103-2753 in the ultraviolet and optical were obtained with the STIS on 2002 July 18 and 20. Three gratings were used: G230L (0'.2 slit width, exposure 2359 s), 


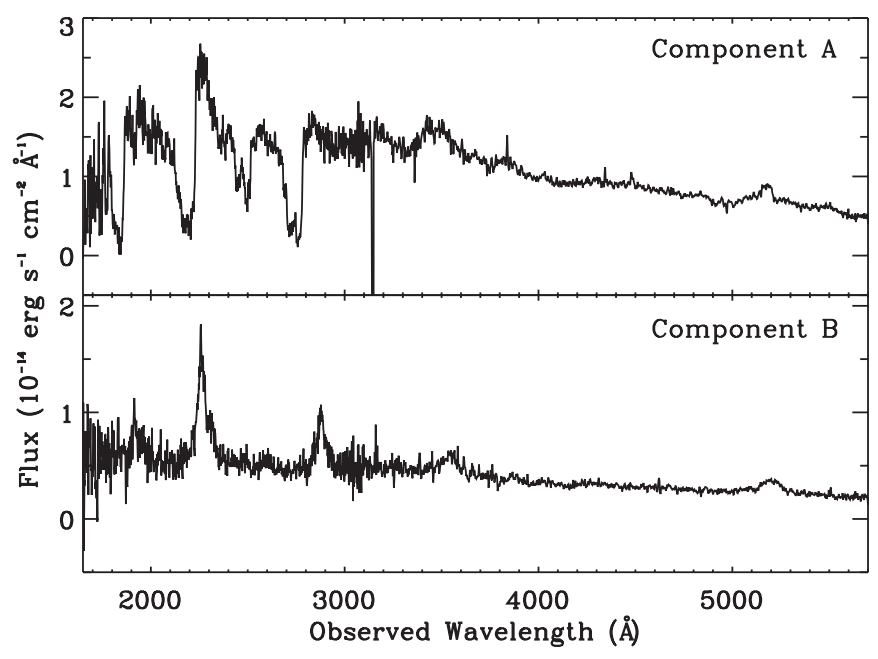

Figure 4. HST STIS spectra of LBQS 0103-2753 for components A and B. See the text for discussion.

G430L(0'.1, $2856 \mathrm{~s})$, and G750L (0'.1, two exposures of $2672 \mathrm{~s}$ and $1834 \mathrm{~s})$. The slit was oriented at a position angle of $150^{\circ}$ to capture both components of the QSO. Figure 4 shows the flux-calibrated spectra from G230L and G430L from the HST pipeline combined into a single spectrum joined at $3100 \AA$. The spectrum shows the prominent BAL features associated with the $\mathrm{O}$ VI, Ly $\alpha$, Si IV, and C IV permitted lines. Comparison with Figure 2 of Junkkarinen et al. (2001) shows little variation in the emission lines or BAL features. The G430L spectrum includes the $\left.\mathrm{C}_{\mathrm{III}}\right]$ and $\mathrm{Mg}$ II lines. The $\mathrm{Mg}$ II BAL remains weak, similar to the case for the 1998 CTIO spectrum obtained by Junkkarinen et al. (2001). This contrasts with the strong Mg II BAL in the LBQS spectrum from 1987 (Morris et al. 1991).

Figure 5 shows a comparison of the spectra of components $\mathrm{A}$ and $\mathrm{B}$ in the $\mathrm{C}$ III] and $\mathrm{Mg}$ II regions. Component $\mathrm{A}$ shows strong emission in Si III $\lambda 1892$ and Al III $\lambda 1857$. Strong Al III emission is common in BAL QSOs (Hartig \& Baldwin 1986). There are also substantial differences between the emission profiles of $\mathrm{Mg}$ II in the two QSOs, including a blueward shift of $\sim 1500 \mathrm{~km} \mathrm{~s}^{-1}$ in the $\mathrm{Mg}$ II peak in component A relative to component B.

The goal of the G750L spectra was to measure the $\mathrm{H} \beta$ profile and detect any narrow [O III] lines that would elucidate the redshift of components A and B. Unfortunately, no [O III] lines were detected. The broad $\mathrm{H} \beta$ lines of both components are detected but too noisy for useful measurements.

\section{DISCUSSION}

Our observations confirm that LBQS 0103-2753 is a dual quasar in an ongoing major merger. This object is distinguished from other known examples by the combination of close spacing, substantial redshift, and high luminosity. Both black holes in this system are quite massive. Junkkarinen et al. (2001) estimated $M_{\mathrm{BH}}=10^{9.0}, 10^{8.5} M_{\odot}$ for components A, B in LBQS $0103-2753$ on the assumption that each component is shining at one-third of the Eddington limit. Alternatively, we may estimate the black hole mass from the broad-line widths. For component $\mathrm{A}$, the $\mathrm{Mg}$ II and $\mathrm{C}$ IV lines are suspect because of the BAL features and velocity shifts. Our HST spectra failed to get good quality detections for the $\mathrm{H} \beta$ line. However, we may use the broad $\mathrm{H} \alpha$ profile from the UKIRT spectra on the assumption that it is dominated by component $\mathrm{A}$. The measured
FWHM of $\mathrm{H} \alpha$ is $4200 \mathrm{~km} \mathrm{~s}^{-1}$. The calibration by Greene \& Ho (2005) then gives an FWHM for $\mathrm{H} \beta$ of $4500 \mathrm{~km} \mathrm{~s}^{-1}$. Using the fit to the continuum flux for component A given by Junkkarinen et al. (2001), we find a continuum luminosity at $\lambda 5100$ rest wavelength of $\lambda L_{\lambda}(5100)=10^{45.77} \mathrm{erg} \mathrm{s}^{-1}$. Using the expression for $M_{\mathrm{BH}}$ given by Shields et al. (2003), we then find $M_{\mathrm{A}}=10^{8.92} M_{\odot}$. For the bolometric luminosity of $10^{46.6} \mathrm{erg} \mathrm{s}^{-1}$ given by Junkkarinen et al. (2001) for component A, this gives $L / L_{\mathrm{Ed}}=10^{-0.4}$. For component $\mathrm{B}$, from the HST spectrum, the FWHM of C IV is $\sim 5200 \mathrm{~km} \mathrm{~s}^{-1}$ and the luminosity at $1350 \AA$ is $v L_{v}(5100) \approx 10^{45.18} \mathrm{erg} \mathrm{s}^{-1}$. Using the expression for $M_{\mathrm{BH}}$ in Vestergaard \& Peterson (2006), we find $M_{\mathrm{BH}} \approx 10^{8.7} M_{\odot}$, giving $L / L_{\mathrm{Ed}}=10^{-0.7}$. (Mg II has an FWHM of $\sim 5700 \mathrm{~km} \mathrm{~s}^{-1}$, similar to C IV, but an unusually flat topped profile. Therefore, we do not use it for $M_{\mathrm{BH}}$ for component $\mathrm{B}$. We caution that there is some controversy regarding the use of $\mathrm{C}$ IV for virial masses of black holes in AGNs.)

We may compare the combined black hole masses for LBQS 0103-2753 to the expected value for the host galaxy luminosity, assuming that this is largely contributed by bulge stars from the progenitor galaxies. The black-hole-bulge luminosity relation is given by Kormendy \& Gebhardt (2001) as $M_{\mathrm{BH}}=\left(10^{7.89} M_{\odot}\right)\left(L_{\mathrm{B}, \text { bulge }} / 10^{10} L_{\odot}\right)^{1.08}$. If we allow $1.5 \mathrm{mag}$ fading from $z=0.8$ to $z=0.0$ (Peng et al. 2006), the present luminosity of the host would be $M_{\mathrm{B}} \approx-21.5$ or $L_{\mathrm{B}} \approx 10^{9.8} L_{\odot}$. The expected black hole mass is then $10^{8.76} M_{\odot}$, compared to $M_{\mathrm{A}}+M_{\mathrm{B}}=10^{9.1} M_{\odot}$ from the above estimates. The scatter in the black hole-bulge relation and the uncertainty in the virial estimates of the black hole masses are each 0.3 dex or more, so these values agree within the uncertainties. We therefore have a picture in which two rather large galaxies with commensurate black holes are being observed at an advanced stage of their merger. The exceptional nature of the present system lies in the vigorous, simultaneous fueling of both black holes, and the lack of obscuration of either nucleus, at least on our line of sight.

Other examples of dual AGNs with good imaging of the host galaxy include COSMOS 100043.15+020637.2 and the $z=0.44$ dual AGN SDSS J1254+0846 separated by $21 \mathrm{kpc}$ (Green et al. 2010). The latter object is more widely spaced than our object, and the tidal tail is more extensive $(\sim 75 \mathrm{kpc})$ and symmetrical. From a suite of numerical simulations of mergers, Green et al. (2010) find a good fit to the observed geometry of SDSS J1254+0846 for a prograde merger on its second pass with a 2:1 baryon mass ratio. From the width of the broad $\mathrm{H} \beta$ line of each component of SDSS J1254+0846, Green et al. derive a black hole mass $M_{\mathrm{BH}}=10^{8.46}, 10^{7.80} M_{\odot}$ for components A, B. This gives Eddington ratios of 0.67 and 0.29 , respectively. The former value and marginally the latter one are higher than typical for AGNs of this luminosity and redshift, which as Green et al. suggest may reflect vigorous fueling in the ongoing merger. Green et al. infer from the merger simulations that the incoming galaxies must have had large disks to produce the large tidal tails and had pre-existing bulges to give the large measured black hole masses at the early merger stage of SDSS J1254+0846. LBQS 0103-2753 involves luminosities and black hole masses one-half to one order of magnitude larger than for SDSS J1254+0846, and similarly high values of $L / L_{\mathrm{Ed}}$.

Comerford et al. (2009) give strong evidence for a dual AGN at $z=0.36$ in COSMOS 100043.15+020637.2. The HST image shows a tidal tail and two bright point sources separated by $1.75 \mathrm{kpc}$, whose spectra are indicative of AGNs. The spacing of the two AGNs and the asymmetrical tidal tail of this object resemble LBQS 0103-2753 more closely than does 


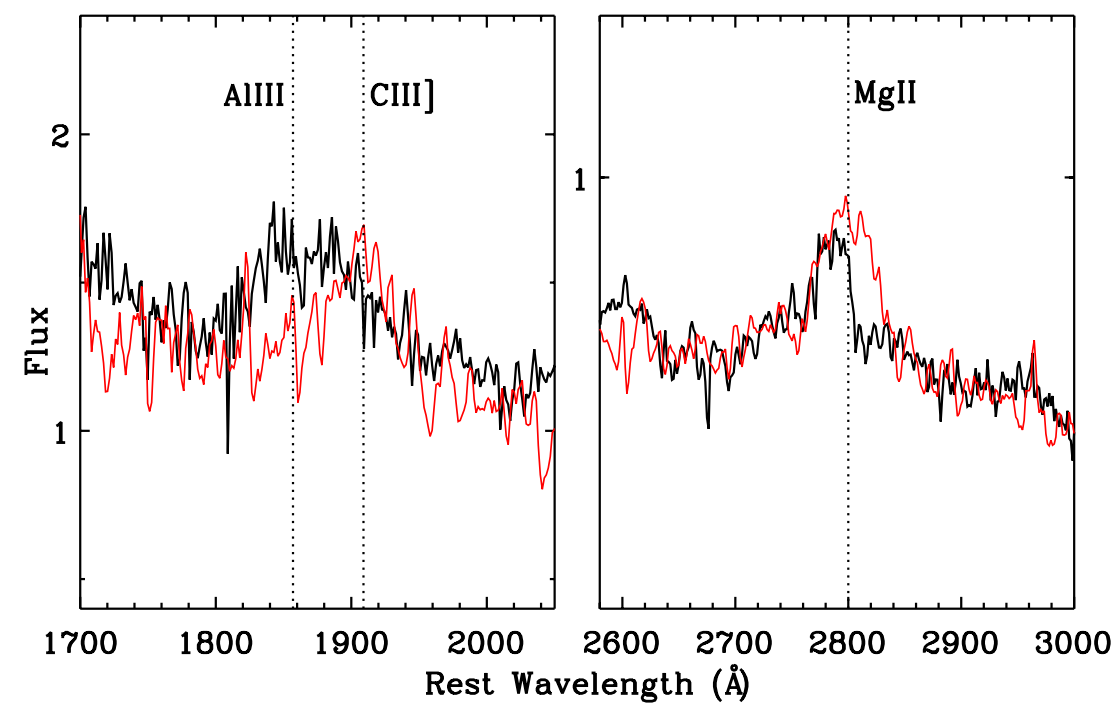

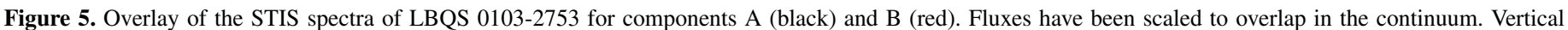

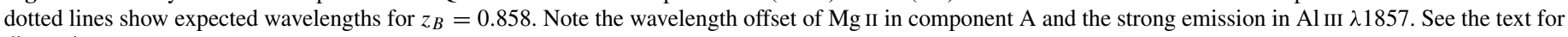
discussion.

(A color version of this figure is available in the online journal.)

SDSS J1254+0846. Civano et al. (2011) present a multiwavelength analysis of this object, giving an optical luminosity for the brighter component of $v L_{v}(5100)=10^{43.9}, 10^{43.6} \mathrm{erg} \mathrm{s}^{-1}$ for the two AGN components, about two orders of magnitude less luminous than LBQS 0103-2753. For the SE (fainter) component, they estimate a black hole mass of $10^{7.8} M_{\odot}$ from the width of the broad $\mathrm{H} \beta$ line. Civano et al. make the interesting suggestion that the SE component may actually be a runaway black hole ejected from the host galaxy nucleus by gravitational radiation recoil or the three-body slingshot mechanism. This interpretation is based in part on a large velocity offset $\left(\sim 1200 \mathrm{~km} \mathrm{~s}^{-1}\right)$ between the broad $\mathrm{H} \beta$ line of the SE component and the narrow emission lines.

Similar in redshift and spacing to LBQS 0103-2753 is the $z=0.71$ dual AGN DEEP2 13025437 (Gerke et al. 2007). This object appears to be in an early-type host with some morphological disturbance. While we cannot analyze the morphology of the host of LBQS 0103-2753 in detail, the large tidal arc and the prominent star formation regions suggest a gasrich merger, consistent with the vigorous fueling required for the two luminous QSOs.

We thank Karl Gebhardt for helpful discussions and assistance. G.S. gratefully acknowledges support from the Jane and Roland Blumberg Centennial Professorship in Astronomy at the University of Texas at Austin.

The United Kingdom Infrared Telescope is operated by the Joint Astronomy Centre on behalf of the science and Technology Facilities of the U.K. Funding for the Sloan Digital Sky Survey (SDSS) has been provided by the Alfred P. Sloan Foundation, the Participating Institutions, the National Aeronautics and Space Administration, the National Science Foundation, the U.S. Department of Energy, the Japanese Monbukagakusho, and the Max Planck Society. The SDSS Web site is http://www.sdss.org/. The SDSS is managed by the Astrophysical Research Consortium (ARC) for the Participating Institutions. The Participating Institutions are The University of Chicago, Fermilab, the Institute for Advanced Study, the
Japan Participation Group, The Johns Hopkins University, the Korean Scientist Group, Los Alamos National Laboratory, the Max-Planck-Institute for Astronomy (MPIA), the Max-PlanckInstitute for Astrophysics (MPA), New Mexico State University, University of Pittsburgh, University of Portsmouth, Princeton University, the United States Naval Observatory, and the University of Washington.

\section{REFERENCES}

Civano, F., Elvis, M., Lanzuisi, G., et al. 2011, ApJ, 717, 209

Comerford, J. M., Griffith, R. L., Gerke, B. F., et al. 2009, ApJ, 702, L82

Comerford, J. M., Pooley, D., Gerke, B. F., \& Madejski, G. M. 2011, ApJ, 737, L19

Foreman, G., Volonteri, M., \& Dotti, M. 2009, ApJ, 693, 1554

Fu, H., Yan, L., Myers, A. D., et al. 2011, ApJ, in press, arXiv:1107.3564

Gerke, B. F., Newman, J. A., Lotz, J., et al. 2007, ApJ, 660, L23

Green, J. E., \& Ho, L. C. 2005, ApJ, 630, 122

Green, P. J., Myers, A. D., Barkhouse, W. A., et al. 2010, ApJ, 712, 762

Hartig, G. F., \& Baldwin, J. A. 1986, ApJ, 302, 64

Hennawi, J. F., Strauss, M. A., Oguri, M., et al. 2006, AJ, 131, 1

Junkkarinen, V., Shields, G. A., Beaver, E. A., et al. 2001, ApJ, 549, L155

Kochanek, C. S., Falco, E. E., \& Muõz, J. A. 1999, ApJ, 510, 590

Komossa, S., Burwitz, V., Hasinger, G., et al. 2003, ApJ, 582, L15

Kormendy, J., \& Gebhardt, K. 2001, in AIP Conf. Proc. 586, 20th Texas Symp. on Relativistic Astrophysics, ed. H. Martel \& J. C. Wheeler (Melville, NY: AIP), 363

Liu, X., Greene, J. E., Shen, Y., \& Strauss, M. A. 2010a, ApJ, 715, L30

Liu, X., Shen, Y., Strauss, M. A., \& Greene, J. E. 2010b, ApJ, 708, L427

Liu, X., Shen, Y., Strauss, M. A., \& Hao, L. 2011, ApJ, 737, L101

Morris, S. L., Weymann, R. J., Anderson, S. F., et al. 1991, AJ, 102, 1627

Peng, C. Y., Impey, C. D., Ho, L. C., Barton, E. J., \& Rix, H.-W. 2006, ApJ, 640, 114

Richards, G. T., Hall, P. B., Vanden Berk, D. E., et al. 2003, AJ, 126, 1131

Richards, G. T., Kruczek, N. E., Gallagher, S. C., et al. 2011, AJ, 141, 167

Rodriguez, C., Taylor, G. B., Zavala, R. T., Philstroöm, Y. M., \& Peck, A. N. 2009, ApJ, 697, 37

Rosario, D. J., McGurk, R. C., Max, C. E., et al. 2011, ApJ, 739, 44

Shields, G. A., Gebhardt, K., Salviander, S., et al. 2003, ApJ, 583, 124

Smith, K. L., Shields, G. A., Bonning, E. W., et al. 2010, ApJ, 716, 866

Stanford, S. A., Sargent, A. I., Sanders, D. B., \& Scoville, N. Z. 1990, ApJ, 349, 492

Vestergaard, M., \& Peterson, B. M. 2006, ApJ, 641, 689

Wang, J., Chen, Y., Hu, C., et al. 2009, ApJ, 705, L76 